\title{
Experiments on bright-field and dark-field high-energy electron imaging with thick target material
}

\author{
Zheng Zhou, ${ }^{1}$ Yingchao Du, ${ }^{1}$ Shuchun Cao, ${ }^{2}$ Zimin Zhang, ${ }^{2, *}$ Wenhui Huang, ${ }^{1, \dagger}$ Huaibi Chen, ${ }^{1}$ \\ Rui Cheng, ${ }^{2}$ Zhijun Chi, ${ }_{1}$ Ming Liu, ${ }^{2}$ Xiaolu Su, ${ }^{1}$ Chuanxiang Tang, ${ }^{1}$ Qili Tian, ${ }^{1}$ Wei Wang, ${ }^{1}$ \\ Yanru Wang, ${ }^{2,4}$ Jiahao Xiao, ${ }^{2,4}$ Lixin Yan, ${ }^{1}$ Quantang Zhao, ${ }^{2}$ Yunliang Zhu, ${ }^{2,4}$ Youwei Zhou, ${ }^{2,4}$ \\ Yang Zong, ${ }^{2}$ and Wei Gai ${ }^{1,3}$ \\ ${ }^{1}$ Department of Engineering Physics, Tsinghua University, Beijing 100084, China \\ ${ }^{2}$ Institute of Modern Physics, Chinese Academy of Sciences, Lanzhou 730000, China \\ ${ }^{3}$ Argonne National Laboratory, Lemont, Illinois 60439, USA \\ ${ }^{4}$ University of Chinese Academy of Science, Beijing 100049, China
}

(Received 4 May 2017; published 12 July 2018)

\begin{abstract}
High-energy charged particle radiography has been used for diagnostics of high-energy density matter, and electrons can serve as a promising radiographic probe that acts as a complement to commonly used proton probes. Here we report on an electron radiography experiment using $45 \mathrm{MeV}$ electrons from an S-band photoinjector, where scattered electrons, after interacting with a sample, are collected and imaged by a quadrupole imaging system. We achieve a spatial resolution of a few microns $(\sim 4 \mu \mathrm{m})$ and a thickness resolution of a few percent for a silicon target of 300-600 $\mu \mathrm{m}$ in thickness. With additional dark-field images captured by selecting electrons with large scattering angles, we show that complementary information for determining external details such as outlines, boundaries and defects can be obtained.
\end{abstract}

DOI: $10.1103 /$ PhysRevAccelBeams.21.074701

\section{INTRODUCTION}

High-energy density physics (HEDP) is the study of matter properties under extreme pressure and temperature conditions, such as those occurring in heavy ion, laser drive fusion and similar processes. Generally, HED matter can only be transiently generated on a timescale of $10 \mathrm{~ns}$ to $1-\mu \mathrm{s}$, and the hydrodynamic response under pressure of $>1 \mathrm{Mbar}$ leads to a high expansion velocity in the range of $\mu \mathrm{m} / \mathrm{ns}$. Therefore, a diagnostic tool with the capability of a picosecond-scale temporal time resolution and a micronscale space resolution is urgently needed. In addition, the diagnostic system must have a large dynamic range and be sensitive to a mixture of high and low $\mathrm{Z}$ (atomic number) components in order to provide an understanding of the hydrodynamics of HED samples. Diagnostics based on charged particle beams have been the subject of studies for many years, beginning with the pioneering efforts of Frank Merrill et al. [1-5]. In recent decades, there has been strong interest in using electron beams to probe the dynamic processes in a target driven by either a laser or heavy ion

\footnotetext{
*zzm@impcas.ac.cn

huangwh@mail.tsinghua.edu.cn
}

Published by the American Physical Society under the terms of the Creative Commons Attribution 4.0 International license. Further distribution of this work must maintain attribution to the author(s) and the published article's title, journal citation, and DOI. beams as well as in online diagnostic for heavy ion induced target damage [6-8]. The process uses a magnetic imaging lens system (quadrupoles or solenoids) that focuses a beam passing through a thick target. The beam is collimated at a focal point and then projected onto a luminescent screen [9]. This method is fundamentally different from typical shadowgraphy with either charged particles or X/gamma rays. Several experiments have been performed with results in good agreement with predictions [10-12], although the spatial resolution has reached only a few tens to $100 \mu \mathrm{m}$, insufficient for a HEDP diagnostics. In general, these experiments required a dynamic process with a nanosecond-scale temporal resolution and a submicron spatial resolution. In the past few decades, the use of rf photocathode techniques has enabled the generation of a pulse train of electrons with high charges $(>n C)$ and high energies $(\sim 100 \mathrm{MeV})$, such as at the Tsinghua Thomson scattering X-ray source [13]. Recently, an experiment using a compact permanent magnet quadrupole based lens was carried out at UCLA, where the micron-scale features of a metal sample were magnified by $\sim 30$ times and imaged with single-shot, low energy ( $4 \mathrm{MeV}$ ) electron bunches [14]. However, the sample used was a thin $(20 \mu \mathrm{m}) \mathrm{Cu}$ target due to the limited penetration power of the low energy electrons, and no areal density resolution was obtained, which is the key criterion for high-energy electron radiographs to be useful for HEDP experiments. Moreover, the targets used in these experiments were seethrough targets instead of opaque thick targets, such as 


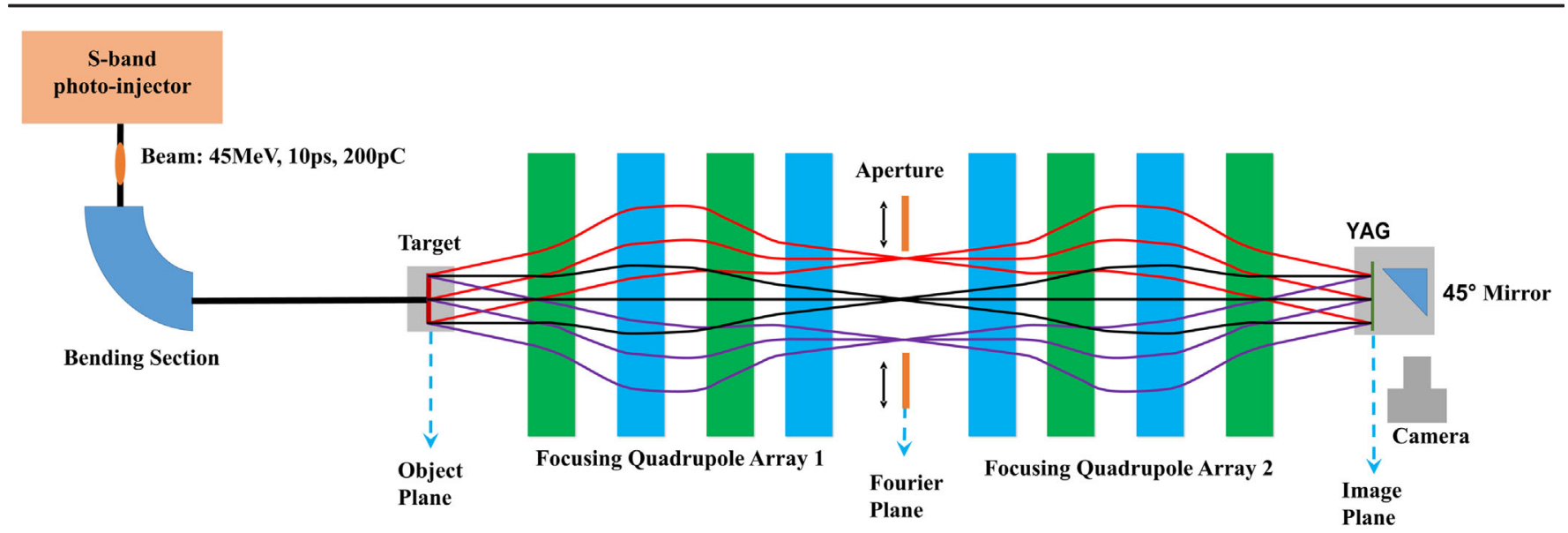

FIG. 1. Experimental setup. The aperture in the vertical or horizontal plane comprises two plates. Each plate is fixed to a step motor and can move independently over a wide range of distances, thereby enabling the selection of on-axis or off-axis particles to form a bright-field or dark-field image, respectively.

those used in our experiment, where we designed and fabricated a set of thick silicon targets with letters etched on in order to replace real high areal density material, in order to check the feasibility of high-energy electron beams from the photoinjector for the HEDP diagnostic.

In this paper, we report on a new experiment performed with photoinjector enabled high-brightness electron beams to obtain single-shot radiographs of a thick target with a micron-order spatial resolution and a few percent thickness resolution. To the best of our knowledge, this is also the first time that dark-field imaging has been achieved in highenergy electron radiography, while similar results have been obtained using a fixed inverse collimator to image thin materials in high-energy proton radiography very recently [15]. This successful demonstration of the imaging of HED targets has proven the feasibility of using high-brightness electron beams for HEDP diagnostics and is expected to have a major impact on HEDP research.

The experimental setup [16] is shown in Fig. 1. The electron beam is produced and accelerated to approximately $45 \mathrm{MeV}$ with an S-band photoinjector and accelerator. The average bunch charge is $200 \mathrm{pC}$, and the bunch duration is $10 \mathrm{ps}$. Detailed parameters of the electron probe hit on the targets are listed in Table I. The electron beam is transported to the target area after a 90-degree bend. After the electron beam passes through the target, the scattered electrons travel through focusing quadrupole arrays which act in a manner analogous to an optical imaging system. An aperture in both the $\mathrm{x}$ and $\mathrm{y}$ planes is located at the focal

TABLE I. Parameters of the electron beams on the target.

\begin{tabular}{lc}
\hline \hline Bunch charge & $200 \mathrm{pC}$ \\
rms normalized emittance & $0.6 \mathrm{~mm} \mathrm{mrad}$ \\
Bunch length & $10 \mathrm{ps}$ \\
rms energy spread & $0.3 \%$ \\
Beam radius on sample & $\sim 2 \mathrm{~mm}$ \\
\hline \hline
\end{tabular}

plane, or the so-called Fourier plane, 1.3-m downstream of the object plane, where the positions of the particles are related only to their initial spread angles. In a commonly used symmetric quadruplet imaging system in high-energy charged particle radiography community, the Fourier planes of $\mathrm{x}$ and $\mathrm{y}$ axes are possibly at the same position. Since the rather wide angle spread after beam passing through the thick targets and the high beam energy, the charge density at the Fourier plane would not be too high to have a big impact on the imaging process. But this problem should be concerned in a low energy imaging scenario where the sample is thin and beam angle is small, such as in the case of Ref. [14], where a triplet was employed instead of a quadruplet to avoid this problem. The electrons selected by the aperture hit a thin $(30 \mu \mathrm{m})$ yttriumaluminum-garnet (YAG) screen on the imaging plane located $2.6-\mathrm{m}$ downstream, and the beam patterns are captured by a CCD detector. The design, optimization and extensive simulation of the whole quadrupolelens-based imaging system were described in Ref. [15]. A brief summary of the parameters of the imaging system is listed in Table II. The main target used in this experiment was an opaque $300-\mu \mathrm{m}$ thick silicon slab engraved with lines of different patterns and depths. A standard 200 mesh molybdenum TEM grid with a hole width of $90 \mu \mathrm{m}$ and a bar width of $35 \mu \mathrm{m}$ was used to determine the spatial resolution of this imaging system. A radiograph of this grid is shown in Fig. 2. Since the thickness of the TEM grid is

TABLE II. Parameters of the quadruplet imaging system.

\begin{tabular}{lccc}
\hline \hline Quads & $\int G d z(\mathrm{~T})$ & Transport matrix element & Value \\
\hline $\mathrm{Q}_{1}$ & 0.315 & $\mathrm{R}_{11}, \mathrm{R}_{33}$ & -2.84 \\
$\mathrm{Q}_{2}$ & -0.743 & $\mathrm{R}_{12}, \mathrm{R}_{34}$ & 0 \\
$\mathrm{Q}_{3}$ & 0.743 & $\mathrm{~T}_{116}, \mathrm{~T}_{126}$ & $6.89,4.81 \mathrm{~m} / \mathrm{rad}$ \\
$\mathrm{Q}_{4}$ & -0.315 & $\mathrm{~T}_{336}, \mathrm{~T}_{346}$ & $6.08,6.01 \mathrm{~m} / \mathrm{rad}$ \\
\hline \hline
\end{tabular}




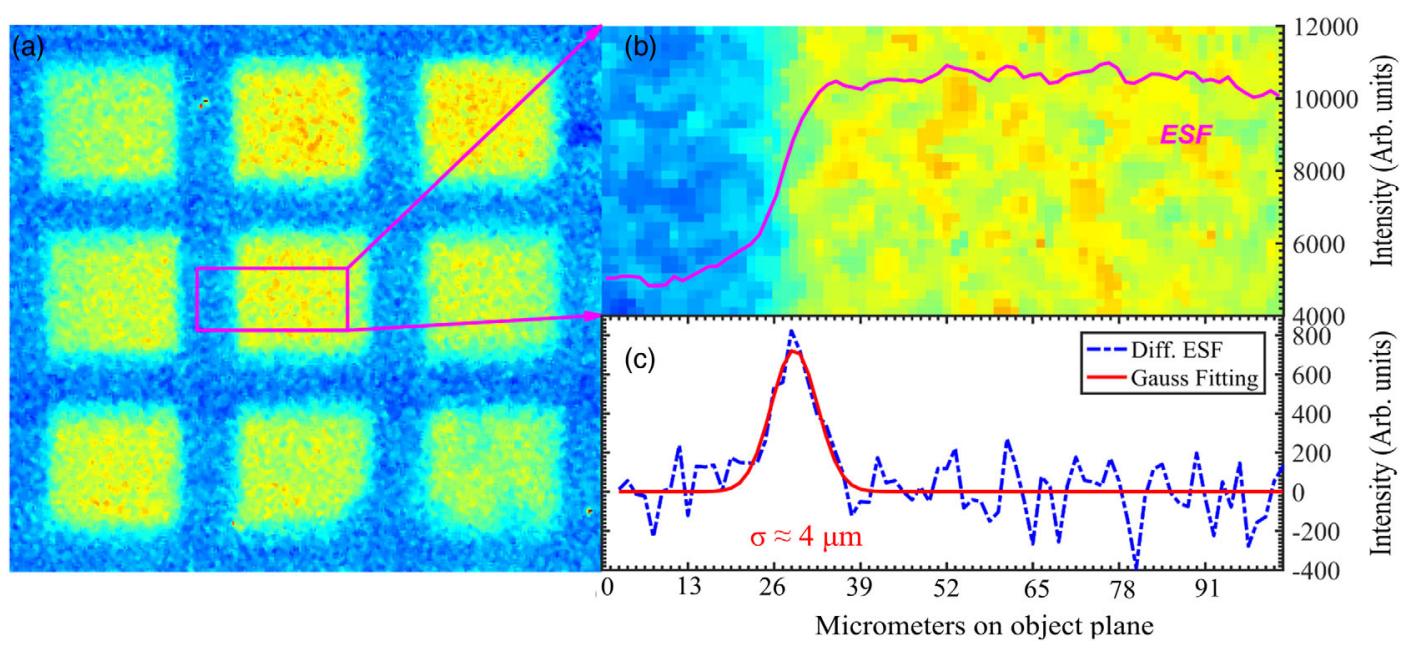

FIG. 2. TEM grid image for spatial resolution calibration. (a) The 200 mesh grid image. (b) Zoom to a small region between the bar and the hole (pink rectangular box) and calculate the intensity projection on the $\mathrm{x}$ axis, i.e., the edge spread function (pink curve). (c) Line spread function and its Gaussian fit, giving a resolution of three pixels (corresponding to about four micros on the object plane).

just about 20 microns, the edge of the bar can be considered as an ideally sharp edge. By studying the step in transmission across the edge of a bar, the edge spread function can be determined, as described in Ref. [10].

The line spread function (i.e., the derivative of the edge spread function) was fitted with a Gaussian function, resulting in a fit rms width of three pixels, on both the horizontal and vertical planes. The good agreement between the data and the fitting results validates the assumption of a Gaussian-shaped line spread function. Given the known geometry of the mesh target, we determined that the spatial resolution on the object plane is about $4 \mu \mathrm{m}$, which is very close to the ultimate resolution limited by the point spread function of the $30-\mu \mathrm{m}$ thick YAG screen.

\section{THICK TARGET IMAGING}

In addition to the detailed spatial resolution measurement, a set of thick targets with etched grooves of a known width and depth were radiographed to study both the space and areal density capabilities of the radiography system, as shown in Fig. 3(a). A logo with the etched letters "HEER" (75 $\mu \mathrm{m}$ in width) was measured experimentally, revealing qualitative surface profile information, as shown in Fig. 3(b). For comparison, we also acquired images of the sample using a scanning electron microscope (SEM), and measured the groove depth at several points with the help of a highresolution profiler.

The letters are clearly visible in the radiographs, but far more detailed features of the logo can also be seen. For example, the nonuniformity of the widths at the junctions of the perpendicular grooves as seen from the radiograph matches well with the SEM surface profile measurement, especially in the cases of the first letter "E" and the letter "R." We also measured four different typical points on the logo as shown in Fig. 3(b). The beam intensity is well correlated with the groove depth, which can be interpreted as a drop in the transmission rate as the effective target thickness increases.

To quantitatively study this system, we concentrated on a small area of a different target etched with the letter "N."

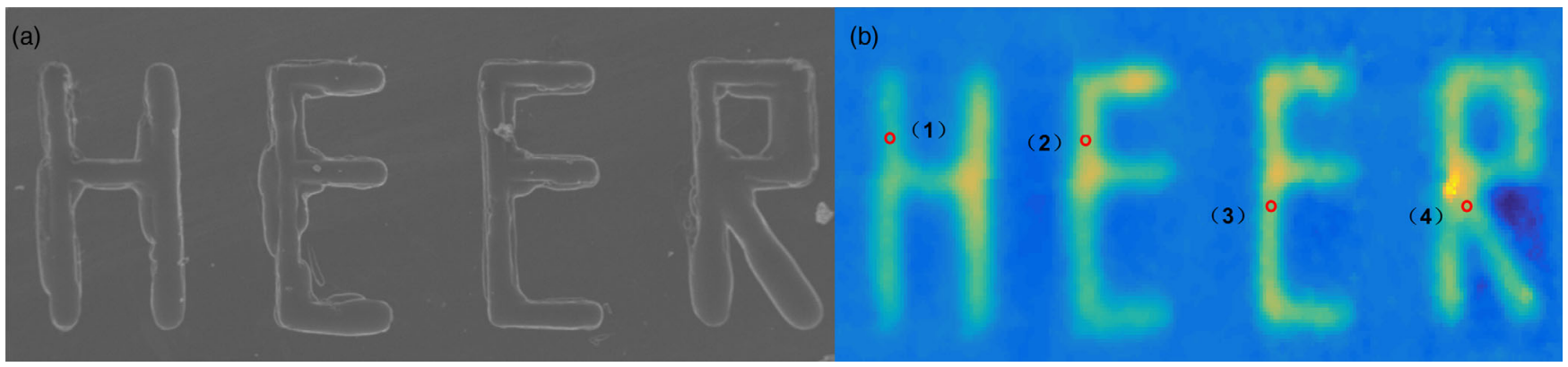

FIG. 3. HEER logo SEM image and radiography image comparison. (a) Profile of the target as scanned with a SEM. Some imperfections of the surface and grooves can be clearly observed. (b) False-color radiographic map of the HEER logo image. The depths of the four spots in (b) (from left to right) were measured to be 42, 50, 46 and $54 \mu \mathrm{m}$, respectively. 

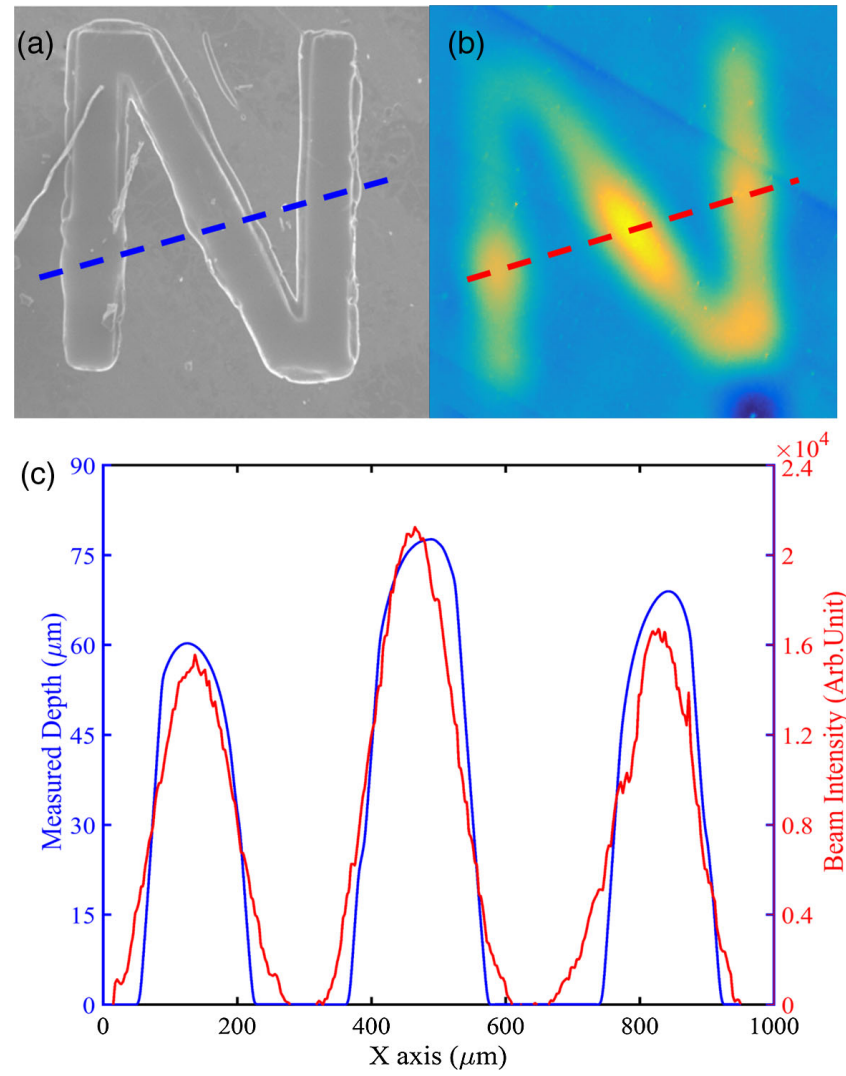

FIG. 4. Letter $\mathrm{N}$ image. (a) SEM surface profile of the letter $\mathrm{N}$ sample under $\times 75$ magnification SEM. The roughness and nonuniformity of the grooves due to the etching process are apparent. (b) Beam intensity distribution radiograph. The signal from the beam passing through the entire target area has been subtracted as background, and the regions with brighter colors correspond to the grooves. (c) Depth information acquired by a high resolution step profiler (blue line) along the red dashed path in (b), compared with the intensity distribution along the same path.

The surface profile of this letter as obtained with the SEM is shown in Fig. 4(a). The beam intensity, as seen in Fig. 4(b), shows a very nonuniform distribution. A depth measurement was performed along the path indicated by the dashed red line. When comparing the depth and intensity distributions, we find that a depth difference of a few tens of microns can be resolved, which is equivalent to a density resolution of a few percent.

\section{DARK-FIELD IMAGING}

Next, we attempted to image the target using the largeangle scattered beam electrons, a process commonly referred to as dark-field imaging, in contrast to on-axis bright-field imaging. This is analogous to the contrastenhancing techniques developed for optical systems, conventional transmission electron microscopes, and $\mathrm{x}$-ray imaging [17]. However, dark-field imaging, or, more generally, scattering-based imaging, using high-energy electrons and exhibiting a good signal-to-noise ratio has not, to our knowledge, been previously demonstrated. Implementing dark-field imaging can provide an additional method of obtaining the desired information about a target, especially when the on-axis information is inaccessible.

In general, radiographic contrast is achieved by the collimation of the electrons through the aperture on the Fourier plane. Considering the beam energy, the main process occurring during beam-target interaction is multiple Coulomb scattering [10], resulting in a Gaussian scattering angle distribution related to the beam energy and target thickness, which is defined by formula (1):

$$
\frac{N\left(l, \theta_{c}\right)}{N_{0}}=\frac{1}{\theta_{0}(l) \sqrt{2 \pi}} e^{-\frac{1}{2}\left(\frac{\theta_{c}}{\theta_{0}(l)}\right)^{2}},
$$

where $\theta_{c}$ is the collection angle of the aperture, and $\theta_{0}$ is defined by

$$
\theta_{0}(l)=\frac{13.6 \mathrm{MeV}}{\beta c P} \sqrt{l}[1+0.038 \ln (l)],
$$

where $P$ is momentum and $l$ is the areal density in unit of the radiation length of the material. For a quasimonoenergetic electron beam, the width of Gaussian distribution is related to the target thickness, with a thicker target having an angular distribution with a larger FWHM, as in the case represented by the blue line in Fig. 5, where $\theta_{0}$ of the thin and thick target is 2 and $6 \mathrm{mrad}$, and responds to $10-\mu \mathrm{m}$ and $70-\mu \mathrm{m}$ thickness silicon target, respectively.

In our study, a 2-mm thick sample holder made of stainless steel with a $3 \mathrm{~mm}$ square hole in the center was used. There was also a step $500 \mu \mathrm{m}$ in width and $400 \mu \mathrm{m}$ in

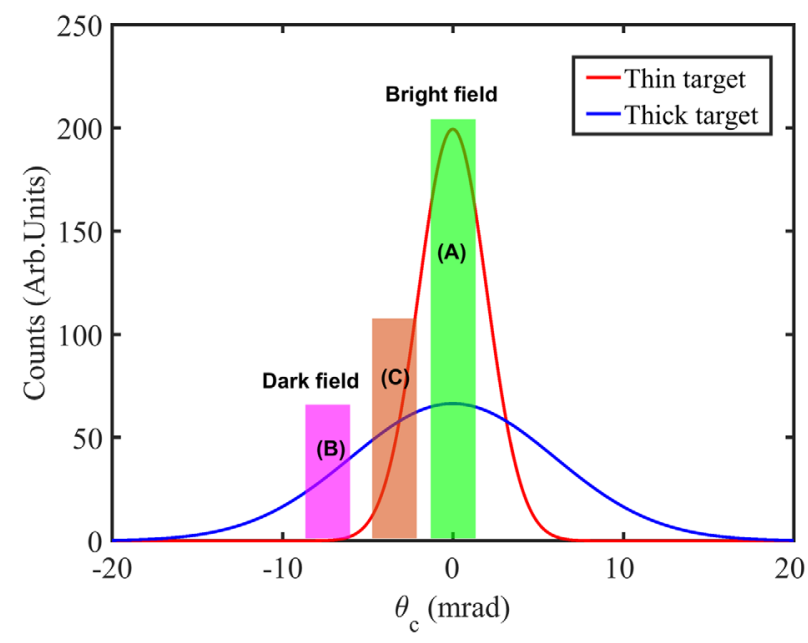

FIG. 5. Principle of dark-field imaging. A probe beam passing through a thicker target will exhibit a wider Gaussian angular distribution. Selecting electrons with larger scattering angles will lead to the formation of a dark-field image (pink region). 
(a)

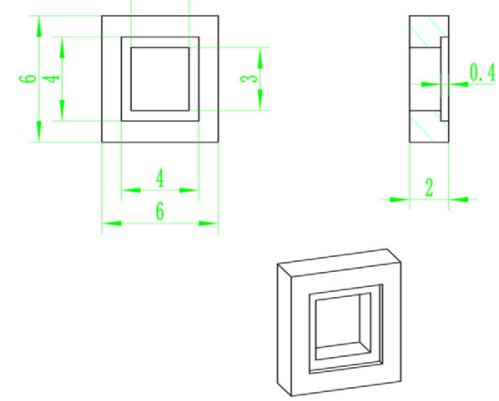

(c)

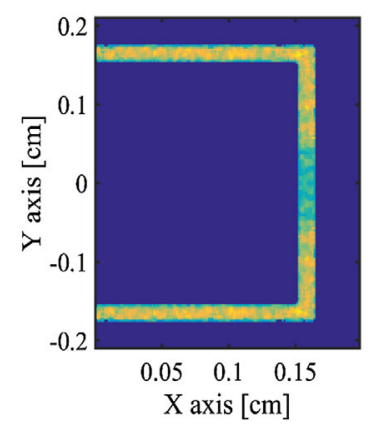

(b)

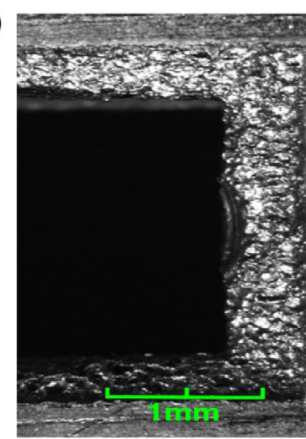

(d)

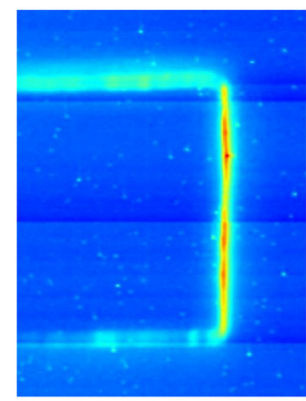

FIG. 6. (a) Sketch of the target. (b) Optical image of half of the hollowed target. (c) Simulated dark-field image. (d) Experimental dark-field image.

height between the hole and the surface, as shown in Fig. 6(a). The detailed features of the right half of the step were observed under a microscope, revealing a visible scratch in the middle part of the right border, as shown in Fig. 6(b). With stepper motors to control location and dimensions of the focus aperture, the aperture was centered on the axis; in this case, as shown in zone (A) in Fig. 5, the ratio of slightly scattered electrons or "unscattered electrons" to all transmitted electrons was much larger than the portion of scattered electrons, as is normally the case of bright-field image. By contrast, when the aperture was placed in an off-axis location, as shown in zone (B) in Fig. 5, only scattered electrons were allowed to pass, leading to the formation of a dark-field image. In practice, the transition from a bright-field image to a dark-field

image can be observed by gradually moving the aperture off the axis. At the position, where the numbers of electrons scattered by a thick target and by a thin target are almost equal, for example in zone (C) in Fig. 5, the contrast generated by multiple Coulomb scattering will be zero, and no distinct radiograph will be seen. A simulated dark-field image is shown in Fig. 6(c), where the real features of the target were included in the simulation. The simulation of the dark-field imaging process is validated by the experimental results, shown in Fig. 6(d). Good agreement with the physical features of the sample is seen in the patterns of the electron images, where the difference in thickness between the hollow region and the edge creates the contrast, and the roughness of the target edge can be observed. This is especially apparent in the case of the visible scratch, where the slight thickness discrepancy induces a nonuniform intensity distribution. We can conclude that dark-field images are also very sensitive to thickness, thus implying a good areal density resolution in dark-field radiography.

For a more quantitative study, we also acquired a darkfield image of a mesh target with one grid square filled in glue [the lighter spot in the bottom left of the grid in Fig. 7(a)], which can be observed through either on-axis bright-field [Fig. 7(b)] or dark-field imaging [Fig. 7(c)]. Here, the resolution of this subtle feature of the target is strongly related to the image contrast, which is defined in terms of the image intensity as $\mathrm{R}=\left(\mathrm{I}_{\max }-\mathrm{I}_{\min }\right)$ / $\left(I_{\max }+I_{\min }\right)$. In the bright-field image, within a small region of three pixels around the "bad" hole, the intensity of each adjacent hole is 3 times brighter than that of the bad hole, resulting in a contrast of 0.5 . This situation is opposite to that of the dark-field image, in which the intensity of the bad hole is 2 times brighter, resulting in a contrast of 0.33 . Although the contrast of the dark-field image is slightly lower, tiny features that cannot be clearly observed in the bright field are more distinctly visible here. Here we note that the whole intensity of the dark-field image is much lower than that of the bright-field image. The dark-field image of the TEM grid in Fig. 7(c) is captured with an exposure time of $1 \mathrm{~s}$, i.e., the image is an accumulation of
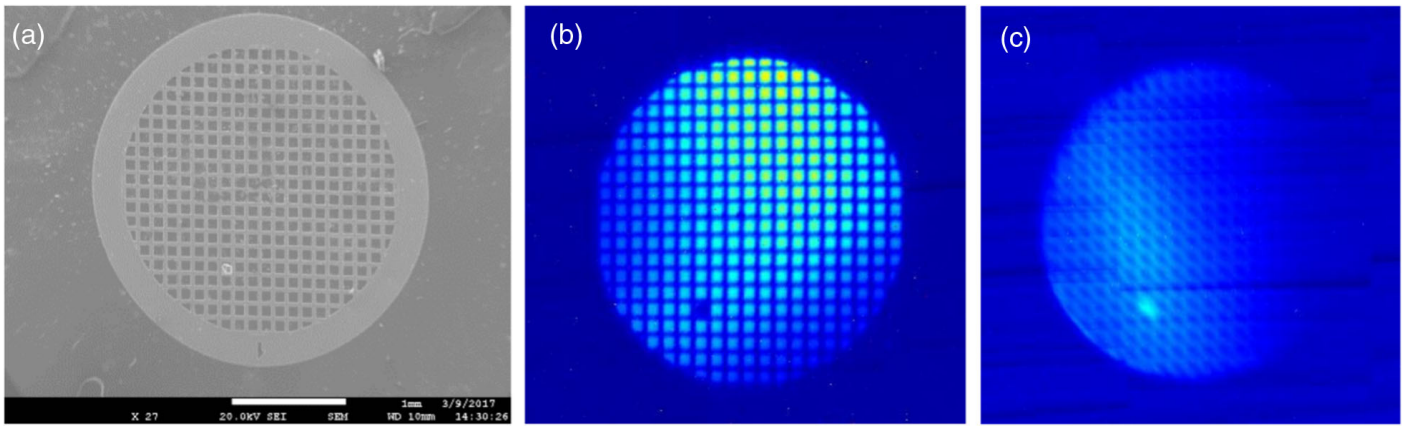

FIG. 7. SEM, bright-field and dark-field images of TEM grid. (a) SEM image of a standard 200 mesh TEM grid. (b) Bright-field image. (c) Dark-field image. 
ten frames. The distortion of the dark-field image may be caused by the large spread angle induced image blurring due to the imperfect point-point imaging system as well as the beam shot-to-shot jitter on the target. Even so, these results illustrate how dark-field imaging can be used for defect identification in the target with a good contrast.

In summary, we have performed high-energy electron radiography on an opaque target with a spatial resolution of $4 \mu \mathrm{m}$ and an areal density resolution of up to a few percent in accuracy. Further resolution improvement could be achieved by optimizing the beam transport system, particularly the magnification factor. Through further magnification by a factor of 10 , a submicron resolution could easily be achieved. The experimental results reported in this article validate this technique as a promising candidate for HEDP or inertial confinement fusion diagnostic. Moreover, we have performed dark-field imaging with the off-axis aperture technique for the first time in high-energy electron radiography experiments, thereby demonstrating that more detailed and complementary information on a specific part of the target can be obtained in addition to that gained through on-axis bright-field imaging and pioneering the use of this advanced imaging technology in the charged particle radiography community.

Furthermore, with the expected picosecond time resolution via picosecond or even femtosecond electron bunches from the existing photocathode $\mathrm{rf}$ gun, this electron imaging system could be used in other dynamic systems, as long as there is a density distribution to probe. This system could even be used for multiple-axis imaging of the target, yielding 3D images [18]. For a target that is much denser or thicker, one could increase the electron beam energy accordingly, with the physics remaining otherwise the same as in this experiment.

\section{ACKNOWLEDGMENTS}

This work was supported by the National Natural Science Foundation of China (NSFC Grant No. 11435015) and the Program of International S\&T Cooperation by the Ministry of Science and Technology of China (Grant No. 2016YFE0104900).

[1] N. King, E. Ables, K. Adams, K. Alrick, J. Amann, S. Balzar, P. Barnes Jr., M. Crow, S. Cushing, J. Eddleman et al., An $800-\mathrm{MeV}$ proton radiography facility for dynamic experiments, Nucl. Instrum. Methods Phys. Res., Sect. A 424, 84 (1999).

[2] S. Kolesnikov, A. Golubev, V. Demidov, S. Dudin, A. Kantsyrev, V. Mintsev, G. Smirnov, V. Turtikov, A. Utkin, B. Sharkov et al., Application of charged particle beams of TWAC-ITEP accelerator for diagnostics of high dynamic pressure processes, High Press. Res. 30, 83 (2010).
[3] Y. M. Antipov, A. Afonin, A. Vasilevskii, I. Gusev, V. Demyanchuk, O. Zyatkov, N. Ignashin, Y. G. Karshev, A. Larionov, A. Maksimov et al., A radiographic facility for the $70-\mathrm{GeV}$ proton accelerator of the institute for high energy physics, Instrum. Exp. Tech. 53, 319 (2010).

[4] P. Rigg, C. Schwartz, R. Hixson, G. Hogan, K. Kwiatkowski, F. Mariam, M. Marr-Lyon, F. Merrill, C. Morris, P. Rightly et al., Proton radiography and accurate density measurements: A window into shock wave processes, Phys. Rev. B 77, 220101 (2008).

[5] C. L. Morris, N. King, K. Kwiatkowski, F. Mariam, F. Merrill, and A. Saunders, Charged particle radiography, Rep. Prog. Phys. 76, 046301 (2013).

[6] F. E. Merrill, Los Alamos National Laboratory (LANL) Technical Report, 2016.

[7] W. Gai, S. Cao, H. Xu, W.-L. Zhan, Z. Zhang, Y. Zhao, J. Qiu, and C. Tang, A high resolution spatial-temporal imaging diagnostic for high energy density physics experiments, in Proceedings of the 5th International Particle Accelerator Conference (IPAC 2014), Dresden, Germany (JACoW, Dresden, 2014), THOAB03, http://jacow.org/ IPAC2014/papers/thoab03.pdf.

[8] W. Gai, J. Qiu, and C. Jing, in Target Diagnostics Physics and Engineering for Inertial Confinement Fusion III (International Society for Optics and Photonics, San Diego, USA, 2014), Vol. 9211, p. 921104.

[9] C. T. Mottershead and J. D. Zumbro, in Proceedings of the Particle Accelerator Conference, Vancouver, BC, Canada, 1997 (IEEE, New York, 1997), Vol. 2, pp. 1397-1399.

[10] F. Merrill, F. Harmon, A. Hunt, F. Mariam, K. Morley, C. Morris, A. Saunders, and C. Schwartz, Electron radiography, Nucl. Instrum. Methods Phys. Res., Sect. B 261, 382 (2007).

[11] F. E. Merrill, C. Morris, K. Folkman, F. Harmon, A. Hunt, and B. King, in Proceedings of the 21st Particle Accelerator Conference, Knoxville, TN, 2005 (IEEE, Piscataway, NJ, 2005), pp. 1715-1717.

[12] Q. Zhao, S. Cao, R. Cheng, X. Shen, Z. Zhang, Y. Zhao, Y.-C. Du, and W. Gai, High energy electron radiography experiment research based on picosecond pulse-width bunch, in Proceedings of the 27th Linear Accelerator Conference, LINAC2014, Geneva, Switzerland (JACoW, Geneva, 2014), Vol. 5, MOPP015, http://jacow.org/ LINAC2014/papers/mopp015.pdf.

[13] C. Tang, W. Huang, R. Li, Y. Du, L. Yan, J. Shi, Q. Du, P. Yu, H. Chen, T. Du et al., Tsinghua Thomson scattering X-ray source, Nucl. Instrum. Methods Phys. Res., Sect. A 608, S70 (2009).

[14] D. Cesar, J. Maxson, P. Musumeci, Y. Sun, J. Harrison, P. Frigola, F. OShea, H. To, D. Alesini, and R. Li, Demonstration of Single-Shot Picosecond Time-Resolved MeV Electron Imaging Using a Compact Permanent Magnet Quadrupole Based Lens, Phys. Rev. Lett. 117, 024801 (2016).

[15] M. S. Freeman, J. Allison, M. Andrews, E. Ferm, J. J. Goett III, K. Kwiatkowski, J. Lopez, F. Mariam, M. Marr-Lyon, M. Martinez et al., Inverse-collimated proton radiography for imaging thin materials , Rev. Sci. Instrum. 88, 013709 (2017). 
[16] Q. Zhao, S. Cao, M. Liu, X. Sheng, Y. Wang, Y. Zong, X. Zhang, Y. Jing, R. Cheng, Y. Zhao et al., High energy electron radiography system design and simulation study of beam angle-position correlation and aperture effect on the images, Nucl. Instrum. Methods Phys. Res., Sect. A 832, 144 (2016).

[17] F. Pfeiffer, M. Bech, O. Bunk, P. Kraft, E. F. Eikenberry, C. Brönnimann, C. Grünzweig, and C. David, Hard-X-ray dark-field imaging using a grating interferometer, Nat. Mater. 7, 134 (2008).

[18] Y. Zhao, Z. Zhang, W. Gai, Y. Du, S. Cao, J. Qiu, Q. Zhao, R. Cheng, X. Zhou, J. Ren et al., High energy electron radiography scheme with high spatial and temporal resolution in three dimension based on a e-LINAC, Laser Part. Beams 34, 338 (2016). 